\title{
THE DIMENSION OF INVERSE LIMITS
}

\author{
M. G. CHARALAMBOUS
}

\begin{abstract}
A result is obtained concerning the dimension of inverse limits of uniform spaces from which several propositions follow that improve earlier results by various authors.
\end{abstract}

1. Introduction. There are two obvious questions concerning inverse limits that arise in dimension theory: (a) What spaces $X$ are inverse limits of "nice" spaces with dimension $\leqslant$ dimension $X$ ? (b) If $X$ is the inverse limit of spaces with dimension $\leqslant n$ (under what conditions) it is true that dimension $X \leqslant n$ ? The first question has been dealt with by Mardešić [9], Pasynkov [15] and Kljušin [8], who generalised the result of Freudenthal asserting that a compact metrisable space $X$ is the inverse limit of a sequence of polyhedra with $\operatorname{dim} \leqslant \operatorname{dim} X$. The second question has received less attention. We have the obvious result for compact spaces, and two results of Nagami, the first asserting that the inverse limit of a sequence of metric spaces of dim $\leqslant n$ has $\operatorname{dim} \leqslant n$ [12], the second that if a countably paracompact space $X$ is the inverse limit of a sequence of normal spaces with $\operatorname{dim} \leqslant n$, then, provided the bonding maps are open and surjective, $X$ is normal and $\operatorname{dim} X \leqslant n$ [13].

In this paper we show that if a uniform space $(X, \mathcal{Q})$ is the inverse limit of uniform spaces $\left(X_{\alpha}, \mathscr{U}_{\alpha}\right)$ with $\mathscr{U}_{\alpha}-\operatorname{dim} X_{\alpha} \leqslant n$, then $\mathscr{U}_{-} \operatorname{dim} X \leqslant n$, where थ - dim is the dimension function introduced by the author [2]. Some corollaries of this result are as follows. (1) The inverse limit of a sequence of perfectly normal spaces with $\operatorname{dim} \leqslant n$ is perfectly normal with $\operatorname{dim} \leqslant n$. (2) If a strongly paracompact space $X$ is the inverse limit of spaces with $\operatorname{dim} \leqslant n$, then $\operatorname{dim} X \leqslant n$. (3) If $X$ is the product of separable metric spaces $X_{\alpha}$ such that any finite product of $X_{\alpha}$ 's has $\operatorname{dim} \leqslant n$, then $X$ has dimension $\leqslant n$. For dimension zero, this result has also been obtained by Morita.

2. Definitions. A subset of a uniform space $(X$, ข) is called थ-open (ขclosed) if it is the inverse image of an open (closed) subset of $R$, the space of real numbers, under a uniformly continuous function. In hyphenated words where " ${ } z$ ", the relativisation of $Q$ to a subset $Z$ of $X$, occurs, we drop " $Z$ ".

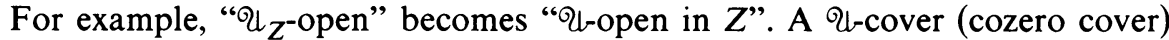
of $X$ is a cover consisting of $d$ is simply a function with domain a class of spaces and range the set

Received by the editors January $25,1975$.

AMS (MOS) subject classifications (1970). Primary 54F45, 54E15; Secondary 54G20.

Key words and phrases. Cozero sets, थ-open sets, dim, थ-dim, inverse limits of spaces, normal, perfectly normal, separable, Lindelöf, pseudocompact, countably compact, and strongly paracompact spaces.

- American Mathematical Society 1976 
$\{-1,0,1,2, \ldots, \infty\}$ such that $d(X)=-1$ if and only if $X$ is empty. To define $d$ it suffices to define " $d(X) \leqslant n$ " for $n=0,1, \ldots$ so that if $d(X) \leqslant n$ and $n \leqslant m$ then $d(X) \leqslant m$, for we can then let " $d(X)=n$ " mean " $d(X) \leqslant n$ and $d(X) \$ n-1$ " and " $d(X)=\infty$ " mean " $d(X) \$ n, n=0,1, \ldots$ ".

थ-dim is defined on the class of all uniform spaces, and dim, dim* on the class of all topological spaces as follows.

थ-dim $X \leqslant n\left(\operatorname{dim} X \leqslant n, \operatorname{dim}^{*} X \leqslant n\right)$ if every finite थ-cover (open, cozero cover) of $X$ is refinable by a finite थ-cover (open, cozero cover) or order $\leqslant n$. (A collection has order $\leqslant n$ if any $n+2$ of its elements have empty intersection.)

As is well known, dim* is the modification of dim introduced by Katětov and Smirnov, and $\operatorname{dim}=\operatorname{dim}^{*}$ for normal spaces. If $\mathcal{U}$ is a metrisable uniformity on $X$, or the topology induced by $\mathcal{Q}$ on $X$ is Lindelöf, or $\mathscr{Q}$ is the uniformity generated by the family of all (bounded) real-valued continuous functions on a topological space $X$, then the cozero sets, and hence $थ-\operatorname{dim} X=\operatorname{dim}^{*} X$. Furthermore, in the first two cases, $X$ is normal and hence $थ-\operatorname{dim} X=\operatorname{dim} X$ [2].

Proofs of the following statements concerning थ-open sets of a uniform space $(X$, थ), which are used implicitly in the sequel, may be found in [2]. The collection of all थ-open (थ-closed) sets of $X$ is closed with respect to finite intersections and countable unions (countable intersections and finite unions). If $G$ is थ-open and $F$ is थ-closed, then $G-F$ is closed. $H$ is थ-open (थ-closed) in a subspace $Y$ of $X$ if and only if $H=G \cap Y$ for some थ-open (थ-closed) set $G$ of $X$. If $E, F$ are disjoint थclosed sets of $X$, there are disjoint थ-open sets $G, H$ of $X$ with $E \subset G$ and $F \subset H$. This will be referred to as the normality property of subsets $A, B$ of $X$ are called distant if for some $U$ in $Q,(A \times B) \cap U=\varnothing$. Proofs of the following results can be found in [3].

Lemma 1. If $A, B$ are distant sets of $(X$, थ), there are distant $थ$-closed sets $E$,

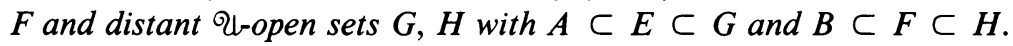

Lemma 2. If $A$ is a थ-closed set of $X$, there are थ-closed sets $A_{k}, k=1,2$, $\ldots$, such that $A=\cap A_{k}$ and $A_{k+1}$ is distant from $X-A_{k}$.

\section{Dimension of inverse limits.}

LeMma 3. Let $(X$, Q $)$ be the inverse limit of an inverse system of uniform spaces $\left(X_{\alpha}, \mathcal{Q}_{\alpha}, f_{\alpha \beta}\right)$ with canonical projections $f_{\alpha}: X \rightarrow X_{\alpha}$, and suppose $A, B$ are distant in $X$. Then for some $\alpha, f_{\alpha}(A), f_{\alpha}(B)$ are distant in $X_{\alpha}$.

Proof. If $g_{\alpha}=f_{\alpha} \times f_{\alpha}$, by [1, Proposition 10, p. 181], for some $\alpha$ and $V$ in $\mathcal{Q}_{\alpha},(A \times B) \cap g_{\alpha}^{-1}(V)=\varnothing$, and hence $\left(f_{\alpha}(A) \times f_{\alpha}(B)\right) \cap V=\varnothing$.

LEMma 4. Let $(X, थ),(X, \mathscr{V})$ be the inverse limits of the inverse systems of uniform spaces $\left(X_{\alpha}, \mathscr{\vartheta}_{\alpha}, f_{\alpha \beta}\right)$ and $\left(X_{\alpha}, \mathfrak{V}_{\alpha}, f_{\alpha \beta}\right)$, respectively, and suppose that the $\mathcal{U}_{\alpha}$-open sets of each $X_{\alpha}$ are precisely its $\Upsilon_{\alpha}$-open sets. Then the $\mathscr{W}_{\text {-open sets }}$ of $X$ are precisely its $\checkmark$-open sets.

Proof. For a given थ-open set $G$ of $X$, let $G_{k}, k=1,2, \ldots$, be थ-open sets such that $G=\bigcup G_{k}$ and $G_{k}$ is distant from $X-G$ (Lemma 2). By Lemma 3, 
for some $\alpha=\alpha(k), f_{\alpha}\left(G_{k}\right), f_{\alpha}\left(X-G_{k}\right)$ are distant in $X_{\alpha}$. Hence there are disjoint $\mathcal{Q}_{\alpha}$-open sets $P_{k}, Q_{k}$ of $X_{\alpha}$ with $f_{\alpha}\left(G_{k}\right) \subset P_{k}$ and $f_{\alpha}(X-G)$ $\subset Q_{k}$ (Lemma 1). By assumption $P_{k}, Q_{k}$ are $\mathfrak{V}_{\alpha}$-open, and since $f_{\alpha}:(X, \mathfrak{V})$ $\rightarrow\left(X_{\alpha}, \mathscr{V}_{\alpha}\right)$ is uniformly continuous $S_{k}=f_{\alpha}^{-1}\left(P_{k}\right), T_{k}=f_{\alpha}^{-1}\left(Q_{k}\right)$ are disjoint $\checkmark$-open sets of $X$ with $G_{k} \subset S_{k}$ and $X-G \subset T_{k}$. Hence $G_{k} \subset S_{k} \subset G, G$ $=\cup S_{k}$ and $G$ is $\checkmark$-open. The converse follows by symmetry.

In the sequel all spaces are invariably assumed to be Tychonoff.

THEOREM. Let $(X, थ)$ be the inverse limit of an inverse system of uniform spaces $\left(X_{\alpha}, \mathscr{\mathcal { U }}_{\alpha}, f_{\alpha \beta}\right)$ with $\mathscr{U}_{\alpha}-\operatorname{dim} X_{\alpha} \leqslant n$ for each $\alpha$. Then $\mathcal{Q}-\operatorname{dim} X \leqslant n$.

Proof. Let $\mathfrak{\mho}_{\alpha}$ be the precompact uniformity on $X_{\alpha}$ whose uniform covers are those that are refinable by the finite $\mathscr{U}_{\alpha}$-covers of $X_{\alpha}$. Then the $\mathfrak{V}_{\alpha}$-open sets of $X_{\alpha}$ are precisely its $\bigcup_{\alpha}$-open sets and

$$
\delta d\left(X_{\alpha}, \mathfrak{V}_{\alpha}\right)=\mathfrak{V}_{\alpha}-\operatorname{dim} X_{\alpha}=\mathscr{\vartheta}_{\alpha}-\operatorname{dim} X_{\alpha} \leqslant n
$$

[2, Proposition 8], where $\delta d$ denotes Smirnov's dimension function; see e.g. [7]. Clearly $\left(X_{\alpha}, \mathfrak{V}_{\alpha}, f_{\alpha \beta}\right)$ is an inverse system of uniform spaces, and if $(X, \mathfrak{V})$ is its inverse limit, applying the inverse limit theorem for $\delta d$ [7, p. 71], we obtain $\delta d(X, \mathfrak{V}) \leqslant n$. Finally, since $\mathfrak{V}-\operatorname{dim} X \leqslant \delta d(X, \mathfrak{V})$ [2, Proposition 7], by Lemma 4, थ $-\operatorname{dim} X=\mathfrak{V}-\operatorname{dim} X \leqslant n$.

Reference to the dimension function $\delta d$ can be completely avoided by applying the obvious result for dim on compact space to the inverse limit of

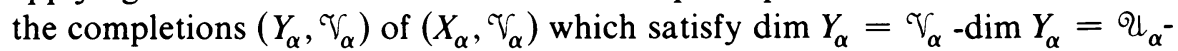
$\operatorname{dim} X_{\alpha} \leqslant n$ [2, Proposition 8], and then appealing to the subset theorem for थ-dim [2, Proposition 3]. Another similar proof can be obtained by using the interesting fact that $\mathcal{Q}-\operatorname{dim} X=\delta d(X, m$ थ $)$, where $(X, m$ थ) is the $\pi$-fine coreflection of $(X, \mathcal{Q})$; see [21]. A uniform space is called $\Re$-fine, or metricfine, if every uniformly continuous function into a metric uniform space remains uniformly continuous when the latter is re-equipped with its fine uniformity. $m$ थ is the coarsest $\Re$-fine uniformity which is finer than $थ$. It can be shown that the precompact reflection of $(X, m$ थ) has as a basis the collection of finite थ-covers of $X$, which implies $\delta d(X, m \mathcal{U})=\mathcal{U}-\operatorname{dim} X$ and also that the $m$ थ-open sets of $X$ are identical with the $थ$-open sets. The same comments apply to the (separable metric)-fine coreflection of [20].

According to Smirnov [16], a Tychonoff space $X$ has the monotonicity property relative to $\operatorname{dim}^{*}$ if $\operatorname{dim}^{*} X \leqslant \operatorname{dim} Y$ for every compactification $Y$ of $X$. If $\mathcal{Q}$ is a uniformity on $X, \mathcal{V}$ is the precompact uniformity on $X$ whose uniform covers are those that can be refined by finite थ-covers, and $(Y, \mathfrak{V})$ is the completion of $(X, \mathfrak{V})$, then $\operatorname{dim} Y=\mathcal{Q}-\operatorname{dim} X$. It follows that $X$ has the monotonicity property relative to $\operatorname{dim}^{*}$ if and only if $\operatorname{dim}^{*} X \leqslant \mathcal{U}-\operatorname{dim} X$ for every uniformity $\mathscr{Q}$ on $X$. The class of all spaces with the monotonicity property relative to dim* contains all strongly paracompact spaces.

Proposition 1. Let $X$ be the inverse limit of an inverse system of Tychonoff spaces $X_{\alpha}$ with $\operatorname{dim}^{*} X_{\alpha} \leqslant n$ for each $\alpha$, and suppose $X$ has the monotonicity property relative to $\operatorname{dim}^{*}$. Then $\operatorname{dim}^{*} X \leqslant n$.

Proof. Let $(X, \mathcal{Q})$ be the inverse limit of $\left(X_{\alpha}, \mathscr{U}_{\alpha}\right)$ where $\mathscr{U}_{\alpha}$ is the Čech 
uniformity on $X_{\alpha}$, i.e. the uniformity generated by the family of all bounded real-valued continuous functions on $X_{\alpha}$. Then $\mathcal{Q}_{\alpha}-\operatorname{dim} X_{\alpha}=\operatorname{dim}^{*} X_{\alpha} \leqslant n$, and by the theorem, $\operatorname{dim}^{*} X \leqslant \mathcal{Q}-\operatorname{dim} X \leqslant n$.

Proposition 2. Let $X$ be the inverse limit of an inverse system $\left(X_{n}, f_{n m}, n\right.$ $\in N)$ of perfectly normal spaces with $\operatorname{dim} X_{n} \leqslant k$ for each $n$ in $N$, the set of natural numbers. Then $X$ is perfectly normal and $\operatorname{dim} X \leqslant k$.

Proof. Let $(X, \mathcal{Q})$ be the inverse limit of $\left(X_{n}, \mathscr{Q}_{n}, f_{n m}\right)$ where $\mathscr{Q}_{n}$ is the Čech uniformity on $X_{n}$. Then $\vartheta_{n}-\operatorname{dim} X_{n}=\operatorname{dim} X_{n} \leqslant k$. If $A$ is closed in $X$, and $f_{n}: X \rightarrow X_{n}$ are the canonical projections, then $A=\bigcap_{n} f_{n}^{-1}\left(\overline{f_{n}(A)}\right)$ [1, Corollary, p, 49]. Since $X_{n}$ is perfectly normal, $\overline{f_{n}(A)}$ is a zero set, and hence a $U_{n^{-}}$ closed set of $X_{n}$. Of course $f_{n}$ is uniformly continuous, and hence $f_{n}^{-1}\left(\overline{f_{n}(A)}\right)$ is थ-closed. Thus $A$ is $थ$-closed, and by the normality property of थ-open sets, $X$ is normal and hence perfectly normal. By our theorem, $\operatorname{dim} X=Q \mathcal{Q}-\operatorname{dim} X$ $\leqslant k$.

That the inverse limit of a sequence of perfectly normal spaces is perfectly normal has also been obtained by Cook and Fitzpatrik [4] by a relatively involved argument.

For the following results we need theorems giving sufficient conditions under which every real-valued continuous function on a subset $Y$ of the product $X$ of spaces $X_{\alpha}$ depends on countably many coordinates, i.e. it is the composite of some real-valued continuous function on a countable subproduct $Z$ of $X_{\alpha}$ 's together with the canonical projection $Y \rightarrow Z$. In Gleason's theorem [7, p. 132], the conditions are that $Y$ is an open set of $X$ and each $X_{\alpha}$ is separable. In Engelking's version of this theorem [5, Theorem 1], it is sufficient to assume that $Y=X$ and that finite products of $X_{\alpha}$ 's are Lindelöf. Noble and Ulmer [19] observe that for Engelking's proof to work it suffices to assume that finite products of $X_{\alpha}$ 's have the property that each uncountable subset has an accumulation point, and this includes the case when finite products of $X_{\alpha}$ 's are countably compact, which is used in the proof of Proposition 7.

Proposition 3. Let $Y$ be an open subset of the product $X$ of separable spaces $X_{\alpha}$ such that any finite product of $X_{\alpha}$ 's has $\operatorname{dim} \leqslant n$. Then $\operatorname{dim}^{*} Y \leqslant n$ in each of the following two cases.

(1) Any countable product of $X_{\alpha}$ 's is hereditarily Lindelöf.

(2) Any finite product of $X_{\alpha}$ 's is perfectly normal.

Proof. $X$ is the inverse limit of finite products $Y_{\beta}$ of $X_{\alpha}$ 's. If $(X, थ)$ is the inverse limit of $\left(Y_{\beta}, \mathscr{Q}_{\beta}\right)$ where $\mathscr{Q}_{\beta}$ denotes the Čech uniformity on $Y_{\beta}$, then for each $\beta, \mathscr{Q}_{\beta}-\operatorname{dim} Y_{\beta}=\operatorname{dim} Y_{\beta} \leqslant n$ and by our theorem $2-\operatorname{dim} X \leqslant n$. By the

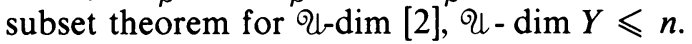

If $f: Y \rightarrow R$ is continuous, by Gleason's theorem [7, p. 132] there is a countable product $Z$ of $X_{\alpha}$ 's and a continuous $g: \pi(Y) \rightarrow R$ such that $f=g \pi / Y$, where $\pi: X \rightarrow Z$ is the canonical projection. If $Z$ is the inverse limit of spaces $Y_{\gamma}$, let $\mathfrak{V}$ be the inverse limit of the corresponding uniformities $\mathcal{U}_{\gamma}$. Then $\pi:(X, \mathscr{Q}) \rightarrow(Z, \mathscr{V})$ is uniformly continuous. Let $H$ be an open set of $R$. In case (1), the cozero set $g^{-1}(H)$ of the Lindelöf space $\pi(Y)$ is $\checkmark$-open in $\pi(Y)$, and hence $f^{-1}(H)=\pi^{-1} g^{-1}(H)$ is -open in $Y$. In case $(2), g^{-1}(H)$ is an 
open set of $Z$, and since any open set of $Z$ is $\mathscr{V}$-open (Proposition 2), $f^{-1}(H)$ is थ-open in $Y$. It transpires that every cozero set of $Y$ is $\operatorname{dim}^{*} Y=\mathcal{Q}-\operatorname{dim} Y \leqslant n$.

Using Engelking's [5] version of Gleason's theorem, we obtain

Proposition 4. Let $X$ be the product of spaces $X_{\alpha}$ every finite product of which has $\operatorname{dim} \leqslant n$. Then $\operatorname{dim}^{*} X \leqslant n$ in each of the following two cases.

(1) Countable products of $X_{\alpha}$ 's are Lindelöf.

(2) Finite products of $X_{\alpha}$ 's are perfectly normal and Lindelöf.

In case (1) of Proposition 4 for dimension 0, since the product theorem for $\operatorname{dim}$ holds when the product is Lindelö [12], we need only assume $\operatorname{dim} X_{\alpha} \leqslant 0$ for each $\alpha$. In this form the result has also been obtained by Morita [11, Theorem 3]. The following result follows from Glicksberg's theorem [7, p. 138].

Proposition 5. If a pseudocompact space $X$ is the product of spaces $X_{\alpha}$, every finite product of which has $\operatorname{dim}^{*} \leqslant n$, then $\operatorname{dim}^{*} X \leqslant n$.

Proposition 6. Let a countably compact space $X$ be the inverse limit of an inverse system of normal spaces and surjective maps $\left(X_{n}, f_{n m}\right)$ with $\operatorname{dim} X_{n} \leqslant k$ for each $n$. Then $X$ is normal and $\operatorname{dim} X \leqslant k$.

Proof. If $E, F$ are disjoint closed sets of $X, f_{n}: X \rightarrow X_{n}$ the canonical projections, then $\bigcap_{n} f_{n}^{-1}\left(\overline{f_{n}(E)} \cap \overline{f_{n}(F)}\right)=\varnothing$, and since $X$ is countably compact, $f_{n}^{-1}\left(\overline{f_{n}(E)} \cap \overline{f_{n}(E)}\right)=\varnothing$ for some $n$. Since each $f_{n m}$ is surjective, so is each $f_{n}[7, \mathrm{p} .72]$ and hence $\overline{f_{n}(E)} \cap \overline{f_{n}(F)}=\varnothing$. It is now clear that since each $X_{n}$ is normal, so is $X$, and if $(X, \mathscr{Q})$ is the limit of $\left(X_{n}, \mathscr{U}_{n}\right)$ where $\mathscr{U}_{n}$ denotes the Čech uniformity on $X_{n}$, then every open $F_{\sigma}$-set of $X$ is थ-open. Our theorem then implies $\operatorname{dim} X=\mathscr{Q}-\operatorname{dim} X \leqslant k$.

Proposition 7. Let $X$ be the product of spaces $X_{\alpha}$ such that any countable product of $X_{\alpha}$ 's is countably compact and any finite product of $X_{\alpha}$ 's is normal with $\operatorname{dim} \leqslant n$. Then $\operatorname{dim}^{*} X \leqslant n$.

Proof. This follows from [19, Proposition 2.1], Proposition 6, and our theorem.

4. Examples. There are various directions in which our results may be conjectured to generalise, and the following examples rule out some of them. They also illustrate the usefulness of these results.

1 . If $X$ is the product of uncountably many copies of $N$, the space of natural numbers with the usual topology, Stone [17] has shown that $X$ is not normal and hence $\operatorname{dim} X>0$. Proposition 3 gives that any nonempty open set of $X$ has $\operatorname{dim}^{*}=0$. According to Morita, S. P. Franklin proved $\operatorname{dim}^{*} X=0$ in 1970.

2. If $R_{\omega}$ is the set of points of the Hilbert space all of whose coordinates are rational, it is well known that $\operatorname{dim} R_{\omega}=1$ and $R_{\omega} \times R_{\omega}=R_{\omega}$ [6]. It follows from Proposition 3 that any nonempty open subset of the product of any number of copies of $R_{\omega}$ has dim* $=1$.

3. For each ordinal $\alpha<\omega_{1}$, the first uncountable ordinal, let $I_{\alpha}$ be a subset of $I^{n}, n$ a fixed element of $\{1,2, \ldots, \infty\}$, such that $0 \in I_{\alpha}$, $\operatorname{dim} I_{\alpha}=0$, for 
$\alpha \leqslant \beta, I_{\alpha} \subset I_{\beta}$, and $I^{n}=\cup_{\alpha<\omega_{1}} I_{\alpha}\left[12\right.$, Theorems 13-15]. Let $M_{\alpha}, M$ be the following subspaces of $\left[0, \omega_{1}\right] \times I^{n}$.

$$
\begin{aligned}
M_{\alpha} & =\bigcup_{\gamma \leqslant \alpha}\{\gamma\} \times I_{\gamma} \cup\left\{(\beta, 0): \beta<\omega_{1}\right\}, \\
M & =\bigcup_{\alpha<\omega_{1}} M_{\alpha}=\bigcup_{\alpha<\omega_{1}}\{\alpha\} \times I_{\alpha} .
\end{aligned}
$$

Define continuous $f_{\alpha \beta}: M_{\beta} \rightarrow M_{\alpha}, \alpha \leqslant \beta$, and $f_{\alpha}: M \rightarrow M_{\alpha}$ by $f_{\alpha \beta}(\gamma, x)$ $=(\gamma, x)$ if $\gamma \leqslant \alpha, f_{\alpha \beta}(\gamma, x)=(\gamma, 0)$ if $\gamma>\alpha, f_{\alpha}(\gamma, x)=(\gamma, x)$ if $\gamma \leqslant \alpha$, and $f_{\alpha}(\gamma, x)=(\gamma, 0)$ if $\gamma>\alpha$. Then $M$ is the inverse limit of the inverse system $\left(M_{\alpha}, f_{\alpha \beta}\right)$ with canonical projections $f_{\alpha}$, and $f_{\alpha}, f_{\alpha \beta}$ are both open and surjective. Yet $M$ is a normal space with $\operatorname{dim} M=n$ [12] while each $M_{\alpha}$ is completely normal with $\operatorname{dim} M_{\alpha}=0$.

4. If $X$ is the inverse limit of an inverse system of normal spaces and open surjective maps $\left(X_{n}, f_{n m}\right)$ with $\operatorname{dim} X_{n} \leqslant k$ for each $N$, is it true that $\operatorname{dim}^{*} X \leqslant k$ ? Nagami's result [13, Theorem 17] asserts that this is so provided that $X$ is countably paracompact. Let $X$ be Michael's hereditary paracompact and Lindelöf space with $\operatorname{dim}=0$ [10], and $Y$ be the irrationals. Then, as Nagami [13] observes, $Y$ is the inverse limit of countable discrete spaces $Y_{i}, i=1,2, \ldots$, and hence $X \times Y$ is the inverse limit of hereditary paracompact and Lindelöf spaces $X \times Y_{i}$ with $\operatorname{dim}\left(X \times Y_{i}\right)=0$ (and open surjective bonding maps). Yet $X \times Y$ is not normal, and hence $\operatorname{dim} X \times Y>0$. An affirmative answer to our question.implies $\operatorname{dim}^{*} X \times Y=0$, and this has been shown to be true [18]. An affirmative answer in fact implies: If $X$ is a normal space and $Y$ is a zero-dimensional separable and completely metrisable space, then $\operatorname{dim}^{*} X \times Y=\operatorname{dim} X$.

5. Can we extend Proposition 4 to arbitrary inverse limits of metric separable spaces? Mrowka is reported to have obtained an example of a closed subspace $X$ of an infinite product of copies of $N$ with $\operatorname{dim}^{*} X>0[14, \S 3]$. Then $X$ is the inverse limit of (zero-dimensional) countable discrete spaces, and yet $\operatorname{dim}^{*} X>0$.

6. If a completely normal first countable space $X$ is the inverse limit of completely normal first countable spaces $X_{\alpha}$ with $\operatorname{dim} X_{\alpha} \leqslant n$, is it true that $\operatorname{dim} X \leqslant n$ ? Example 3 shows that this is false if we drop the assumption that $X$ is completely normal. Let $A$ be a subset of a completely normal first countable space $X$, and for each $x$ in $X-A$, let $A_{x}=X-\{x\}$. Then the normal space $A_{x}$ is the countable union of closed sets with $\operatorname{dim} \leqslant \operatorname{dim} X$ and hence $\operatorname{dim} A_{x} \leqslant \operatorname{dim} X$. Since $A$ is the inverse limit of the cofinite subsets of $X$ containing $A$, if the answer to our question is affirmative, then the subset theorem for completely normal first countable spaces is valid. The latter is an open question in dimension theory.

\section{REFERENCES}

1. N. Bourbaki, Elements of mathematics. General topology. Part 1, Hermann, Paris; AddisonWesley, Reading, Mass., 1966. MR 34 \# 5044a.

2. M. G. Charalambous, A new covering dimension function for uniform spaces, J. London Math. Soc. 11 (1975), 137-143.

3. - Uniformity-dependent dimension functions (to appear). 
4. H. Cook and B. Fitzpatrick, Jr., Inverse limits of perfectly normal spaces, Proc. Amer. Math. Soc. 19 (1968), 189-192. MR 36 \#3306.

5. R. Engelking, On functions defined on Cartesian products, Fund. Math. 59 (1966), 221-231. MR 34 \# 3546.

6. W. Hurewicz and H. Wallman, Dimension theory, Princeton Math. Ser., vol. 4, Princeton Univ. Press, Princeton, N.J., 1941. MR 3, 312.

7. J. R. Isbell, Uniform spaces, Math. Surveys, no. 12, Amer. Math. Soc., Providence, R.I., 1964. MR 30 \#561.

8. V. L. Kljušin, Perfect mappings of paracompact spaces, Dokl. Akad. Nauk SSSR 159 (1964), 734-737 = Soviet Math. Dokl. 5 (1964), 1583-1586. MR 30 \#3447.

9. S. Mardešić, On covering dimension and inverse limits of compact spaces, Illinois J. Math. 4 (1960), 278-291. MR 22 \#101.

10. E. A. Michael, The product of a normal space and a metric space need not be normal, Bull. Amer. Math. Soc. 69 (1963), 375-376. MR 27 \#2956.

11. K. Morita, On the dimension of the product of Tychonoff spaces, General Topology and Appl. 3 (1973), 123-133. MR 47 \#9574.

12. K. Nagami, Dimension theory, Pure and Appl. Math., vol. 37, Academic Press, New York and London, 1970. MR 42 \#6799.

13. Countable paracompactness of inverse limits and products, Fund. Math. 73 (1971/72), no. 3, 261-270. MR 46 \#844.

14. P. Nyikos, Prabir Roy's space $\Delta$ is not $N$-compact, General Topology and Appl. 3 (1973), 197-210. MR 48 \#3007.

15. B. Pasynkov, w-mappings and inverse spectra, Dokl. Akad. Nauk SSSR 150 (1963), 488-491 = Soviet Math. Dokl. 4 (1963), 706-709. MR 27 \#2955.

16. Ju. M. Smirnov, On the dimension of increments of bicompact extensions of proximity spaces and topological spaces. II, Mat. Sb. 71 (113) (1966), 454-482; English transl., Amer. Math. Soc. Transl. (2) 84 (1969), 219-251. MR 35 \# 4887.

17. A. H. Stone, Paracompactness and product spaces, Bull. Amer. Math. Soc. 54 (1948), 977-982. MR 10, 204.

18. J. Terasawa, On the zero-dimensionality of some non-normal product spaces, Sci. Rep. Tokyo Kyoiku Daigaku Sect. A 11 (1972), 167-174. MR 46 \#9948.

19. N. Noble and Milton Ulmer, Factoring functions on Cartesian products, Trans. Amer. Math. Soc. 163 (1972), 329-339. MR 44 \#517.

20. Z. Frolik, A note on metric-fine spaces, Proc. Amer. Math. Soc. 46 (1974), 111-119.

21. A. W. Hager, Some nearly fine uniform spaces, Proc. London Math. Soc. (3) 28 (1974), 517-546.

Department of Mathematics, Ahmadu Bello University, Zaria, Nigeria 